\title{
Critical Factors to Practicing Medical Acupuncture in Family Medicine: Patient and Physician Perspectives
}

\author{
Christy J. W. Ledford, PhD, Carla L. Fisher, PhD, David A. Moss, MD, and \\ Paul F. Crawford, III, MD
}

Introduction: Although an increasing number of physicians are completing medical acupuncture training, only half of those physicians are able to successfully incorporate acupuncture into practice. We conducted a qualitative study to identify the factors and barriers that can enhance and impede physicians' delivery of and patients' engagement in medical acupuncture within the family medicine clinic.

Methods: We conducted interviews with 15 family physicians and 17 patients in a US family medicine clinic that has integrated medical acupuncture into its practice. Audio recordings were transcribed and analyzed by 2 members of the study team in ATLAS.ti, using the constant comparative method.

Results: We identified 3 physician themes of factors/barriers that impact delivery/engagement: 1) patients' aversion to needles, 2) time challenges, and 3) access to resources. We identified 3 additional themes from patient interviews: 1) appointment access; 2) wanting noninvasive, no side effects alternative; and 3 ) openness to anything.

Discussion: The factors and barriers reported by physicians/patients help illustrate conditions needed to enhance physicians' ability to provide acupuncture and patients' willingness to engage and sustain it. Participants' experiences help to illustrate strategic approaches to managing these barriersstrategies that can be used by other individuals/institutions to enhance care delivery and patient engagement. (J Am Board Fam Med 2018;31:236-242.)

Keywords: Acupuncture, Family Physicians

Acupuncture enhances the quality of life for patients in the treatment of chronic and acute pain, improving both mental and physical health. ${ }^{1}$ Research demonstrates benefits associated with chronic pain ${ }^{2}$, low-back and pelvic pain associated

This article was externally peer reviewed.

Submitted 19 September 2017; revised 16 November 2017; accepted 28 November 2017.

From the Department of Family Medicine, F. Edward Hebert School of Medicine, Uniformed Services University of the Health Sciences, Bethesda, MD (CJWL); College of Journalism \& Communications, STEM Translational Communication Center, University of Florida (UF) Health Cancer Center; UF Center for Arts in Medicine, University of Florida, Gainesville, FL (CLF); Nellis Air Force Family Medicine Residency Program, Mike O'Callaghan Military Medical Center, Nellis Air Force Base, NV (DAM, PFC). dation.

Funding: This work was supported by the Acus ${ }^{\mathrm{TM}}$ Foun-

Disclaimer: The opinions and assertions contained herein are the private views of the authors and are not to be construed as official or as reflecting the views of the US Air Force, the Uniformed Services University of the Health Sciences, or the Department of Defense at large. with pregnancy ${ }^{3}$, migraine headaches ${ }^{4}$, and cancerrelated fatigue. ${ }^{5}$ Medical acupuncture is a growing field that can provide successful treatment for patients coping with chronic and acute mental and physical ailments. ${ }^{6}$ At its foundation, medical acupuncture is the therapeutic insertion of solid needles in various combinations and patterns. ${ }^{7}$ Medical acupuncture encompasses French energetic acupuncture, trigger point acupuncture, and Worlsey 5-element acupuncture ${ }^{8}$ as well as Chinese scalp acupuncture, comprehensive auricular acupuncture, and electroacupuncture.

As a discipline, medical acupuncture is acupuncture that has been integrated into medical practice. Medical acupuncture allows physicians to diagnose

Corresponding author: Christy J. W. Ledford, PhD, Uniformed Services University of the Health Sciences, 4301 Jones Bridge Road, Bethesda, MD 20814 (E-mail: christian.ledford @usuhs.edu). 
and treat Western diagnoses with Western treatments and to add acupuncture as an adjunct without the requirement of a referral to an acupuncturist. $^{9-11}$ As evidence establishes the efficacy of medical acupuncture ${ }^{12}$ and more physicians receive medical acupuncture training, primary-care clinics need to know how to most effectively integrate it into practice.

Ten years ago, Yeh, Ryan, Phillips, and Audette $^{13}$ demonstrated that although an increasing number of physicians were completing medical acupuncture training, only half of those physicians were able to successfully incorporate acupuncture into practice, citing issues related to time and money. Time constraints are especially salient in medical acupuncture in which physicians are adding procedural treatments within the confines of a primary-care appointment. Adding an acupuncture treatment can take 10 to 90 minutes. ${ }^{14}$ However, little else is known about other factors impacting acupuncture integration into medical practice from both physicians' and patients' experiences.

\section{Methods}

To develop a better understanding of how the factors/barriers can enhance/impede physicians' delivery of and patients' engagement in medical acupuncture within the family medicine clinic, we conducted a qualitative study using semistructured interviews with physicians and patients. The study was approved by the Wilford Hall Institutional Review Board. Data were collected between June 2016 and January 2017.

\section{Participants}

Purposive sampling included physicians who had completed a 300-hour medical acupuncture course and patients who had received at least 1 acupuncture treatment in the clinic. Research coordinators recruited 15 family physicians and 17 patients ( $\mathrm{n}=$ 32) from a family medicine clinic in a Western US medical center. At this medical center, of the 48 family physicians who provide patient care, 17 $(35.4 \%)$ are fully trained in medical acupuncture. See Tables 1 and 2 for demographics of participants.

\section{Procedures}

Local research coordinators consented participants and collected demographics. The first 2 authors (trained experts in qualitative research) conducted
Table 1. Physician Demographics $(n=15)$

\begin{tabular}{lc}
\hline Gender [n, (\%)] & \\
Female & $3(20 \%)$ \\
$\quad$ Male & $12(80 \%)$ \\
Years practicing medicine & Mean, $3.73(\mathrm{SD}, 1.94)$ \\
Years practicing medical acupuncture & Mean, 2.00 (SD, 1.31) \\
\hline
\end{tabular}

$\mathrm{SD}$, standard deviation.

semistructured, in-depth interviews in a private clinic room. Participants described factors/barriers enhancing/impeding delivery and/or engagement. Interviews averaged 1 hour. Professional transcriptions included 812 pages of data. For confidentiality, a coding system was employed (eg, DR1 = physician; PT1 = patient).

\section{Analysis}

Using the constant comparative method ${ }^{15}$, data were thematically analyzed separately by group and compared to maintain sensitivity to variant experiences. To become immersed in the data, the first 2 authors met after each interview to discuss findings and note emergent patterns to maximize saturation. They also reviewed the transcripts in full multiple times before beginning analysis. A coding manual was developed and continuously revised to establish a set a themes with defining characteristics (properties) as data were collected. Data analysis was concurrent with data collection and included

Table 2. Patient Demographics $(n=17)$

\begin{tabular}{lr}
\hline Age (years) & Mean, 52.18 (SD, 13.93) \\
Gender [n, (\%)] & $10(58.8 \%)$ \\
Female & $7(41.2 \%)$ \\
Male & \\
Number of acupuncture treatments & \\
$\quad$ received [n, (\%)] & $4(23.5 \%)$ \\
Low utilizer (up to 5 treatments) & $9(52.9 \%)$ \\
Medium utilizer (6 to 20 & \\
$\quad$ treatmnets) & $4(23.5 \%)$ \\
High utilizer (>20 treatments) & \\
Primary reason for receiving & \\
$\quad$ acupuncture treatment ${ }^{*}[\mathrm{n},(\%)]$ & $(47.1 \%)$ \\
Chronic pain & \\
Musculoskeletal injury or pain & $5(29.4 \%)$ \\
Stress/anxiety & $4(23.5 \%)$ \\
\hline
\end{tabular}

*List indicates primary reason for receiving acupuncture. Multiple patients reported receiving acupuncture for more than one reason.

${ }^{\dagger}$ Includes persistent migraine.

$\mathrm{SD}$, standard deviation. 
memo-keeping of emergent themes and meetings to discuss findings, develop patterns, enhance reflexivity, and attain saturation. These verification techniques helped ensure trustworthiness of the data (ie, reliability and validity).

Using ATLAS.ti (Berlin, Germany, Scientific Software Development $\mathrm{GmbH}$ ) as a data management tool, the analytic steps of Strauss and Corbin ${ }^{16}$ were used: 1 ) discovery of concepts, code assignment; 2) grouping related concepts for categories; and 3) characterizing theme dimensions for thick description. Special attention was paid to interrelationships between themes for deeper conceptualizations using the insiders' own words. Themes presented here were reported by at least $70 \%$ in each group.

\section{Results}

Findings are separated by group to highlight unique and overlapping experiences. Theme dimensions and interrelationships are noted providing an iterative conceptualization to illustrate a deeper understanding of how barriers/factors functioned. Findings include how physicians/patients strategically contended with barriers providing translational value for integrating acupuncture into Western practice.

\section{Physician Perspectives}

The following factors/barriers impacted delivery/ engagement: 1) patients' aversion to needles, 2) time challenges, and 3) access to resources.

\section{Patients' Aversion to Needles}

Two theme dimensions accounted for patients' hesitancy: 1) prior experience with Battlefield Acupuncture (BFA), and 2) fear of needles. Physicians believed patients who had BFA (auricular acupuncture with aiguille d'acupuncture semi-permanente, translated to English as semipermanent needle) were resistant due to pain and low success. As 1 physician reported:

[BFA needles] do pack much more of a punch ... When they look at the studies to see how correct these points [were]-If somebody got trained in BFA for a 6-hour course and they see what they are doing a couple months later-it was very, very off-the points. A lot of times, I feel like the people who got BFA are like, well, you did not get the points, probably not in the best place, and then you got these painful needles, and it stuck around for 3 or 4 days ... That really turned them off to acupuncture in general. (DR11)

Patients' needle aversion varied, impacting willingness differently. Patients with extreme phobia were not pursued. A physician explained, "For those you just have to say, 'This is not for you. It is just not for everyone.' If they are going to have a panic attack in the moment... it is not therapeutic at all" (DR7). To reduce aversion, physicians used 2 strategies sometimes concurrently: 1) demonstrating painlessness, and 2) using a simple treatment. A physician explained:

There's different levels of treatment. Some are really simple ones. . . . If they are a little on edge, I always try something very simple that does not really hurt, it is very relaxed, and people barely ever notice those needles. Once they do that, they tend to do well, especially people who pass out with needles.... That is the problem with BFA. It is a big pipe that someone shoves in your ear that hurts! (DR1)

\section{Time Challenges}

Time challenges included 3 dimensions: 1) treatment, 2) patient, and 3) physician constraints. First, treatment time was needed. Acupuncture was not successful with patients wanting a "quick fix" not consecutive treatments. Second, patients' constraints (eg, travel/transportation limits) inhibited the time needed (multiple appointments) for acupuncture success. Lastly, appointment time hindered physicians' ability to fully explain or practice acupuncture, as described below:

We have 20 medical appointments ... You have to guard yourself. If you do [acupuncture] for everyone... you are going to end up really far behind in clinic. At the end of the day, my wife really likes to see me at home too.... It is still tough to provide what I consider to be good care and do acupuncture all in 1 appointment ... It is a tough time pyramid. I do feel strained and sometimes pushed and torn in that respect. (DR6)

[If] you are doing a follow-up for med refills and going through any of their chronic problems, you really do not have time to go through an acupuncture treatment as well. It must al- 
most be, unless it is in line with what they are coming in for, a separate appointment. (DR7)

Physicians often required a second appointment to initiate treatment. This strategy addressed physicians' constraints but not patients'. Physicians still described challenges given that treatments warranted 20 minutes plus interaction time (especially with needle aversion). This was further complicated if patients arrived late or physicians lacked technician support and space. The subsequent theme illustrates this further.

\section{Access to Resources}

Three types of resources were critical to delivery: 1) personnel/technical support, 2) equipment/ space, and 3) leadership support. Appointments were limited given a lack of trained physicians further complicated with a residency program and multiple duties in family medicine. This tied to time barriers. Yet, as seen below, physicians reported having technical support helped them strategically combat these limitations:

I have 2 technicians at all times. Everyone else in the clinic has 1 . They threatened to take me down to 1 . I threw a hissy fit and you have to to get anything done around here. ... Your technicians will make and break you, and they will allow you to do acupuncture or not. I have my technicians trained to increase the electricity ... turn over the rooms with blankets, pillows ... set up all the electric PENS [percutaneous electrical nerve stimulation] unit and the wires on either side ... and get [patients] ready to go. . . . When they are done, they will take them all out correctly and roll everything up. (DR4)

Related to this was having space/equipment. These physicians usually reported having the necessary equipment/supplies (needles, pillows, stimulator machines); however, space (comfortable, stocked rooms) was limited. This coincided with time constraints:

I have 2 rooms . . o only 20 minutes per appointment. [Patients are] going to be in there from 20 minutes after I place the needles and now I've spent 30 minutes. I need them to be somewhere that they can sit, and I can continue with my flow of patients.... We're all looking for the extra room. That is the biggest key to not being able to do it is space. (DR7)

One physician went "rogue," stocking 1 room for acupuncture and using 4 rooms, enabling him to treat 2 patients simultaneously.

Lastly, physicians stressed that support from hospital administration was imperative. Many expressed concerns that without leadership support, they would not be able to continue acupuncture when changing to a new practice locations:

My biggest concern is not really with our program here but just what is going to happen [when we leave]. ... How do they apply this and how do they get protection to the extent that their [hospital leadership] and their chief of medical service understand the value of this and support them in making this a reality across the [system]? (DR15)

Physicians reported that this "buy-in" was a precursor to getting acupuncture adopted across the health system, which many advocated for:

To really make an impact, to get the [system leaders] to care about acupuncture, you need to start getting commanders to come out and talk about what the benefit is. And not hospital [leadership]—you need to get operational commanders . . . [to say], "Hey, this is really impacting us in a positive way. I want more acupuncturists to be able to provide care to my pilots." ... If we can wedge our way into that community, to where we can make a significant impact, that is where it is really going to give us some legitimacy.... That is the realm we need to head to be able to get legitimacy in the military as a whole. (DR16)

\section{Patient Perspectives}

Patients acknowledged similar experiences as physicians (needle fear, time) but highlighted different issues: 1) appointment access; 2) wanting noninvasive, no-side-effects alternative; and 3) openness to anything.

\section{Appointment access}

Patients encountered scheduling challenges related to 1) systematic and 2) organizational constraints. First, appointments were scarce, limiting options and impacting patients' ability to sustain treatment. Patients noted the demand outweighed the number 
of trained physicians but also described system challenges like location (treatment offered at 1 clinic location). This mostly impacted patients traveling over an hour. One patient reported that long travel exacerbated his ailment and resulted in his 1 of 2 days off of work being solely for appointments. Other patients described systematic difficulty with referrals. Patients sometimes seemed confused about when they needed a referral but reported delays or not seeking the treatment because of the time needed for a referral. Patients reported organizational issues even when being very proactive themselves: 1) no one answered the phone or they were redirected (given the "run-around"); and 2) physicians' calendar was unavailable (posted 1 month in advance).

Because of systematic/organizational barriers, patients experienced long lapses between treatments. As one stated, "My back does not wait 3 to 4 weeks" (PT3). They sought other treatments (narcotics, paying for outside chiropractors). To contend with these barriers and obtain access, patients employed multiple strategies as this patient described:

As soon as I come out [of appointment], I go straight to the window and ask them if the calendar's out and start booking them. ... I try and book as many as I can to make sure that they form a sequence of 2 weeks. . . The phone is not always answered or does not go through. You are better off seeing people face to face to make an appointment. . . . It is not the regular way of making appointments. You usually call central appointments, and then they usually go ahead and find something for you. No-they redirect you to this actual clinic for them to actually schedule the appointment. If nobody's answering or if the number drops or whatever, you cannot get an appointment. (PT11)

\section{Wanting Noninvasive, No-Side-Effects Alternative}

Many patients had seen many specialists for chronic ailments/injuries and typically given 2 options they wanted to avoid/limit: 1) surgery and/or 2) narcotics. Patients were motivated to try acupuncture to avoid surgery for various reasons (surgery did not work or exasperated the ailment; surgery would not definitely eliminate pain; timing issues, and long recovery; invasiveness). Patients were also motivated to stop/avoid narcotics due to side effects and to enhance current wellbeing as this woman described:

[My doctor] said about being pain free can make you so much a happier person. [Patient laughs] I do not know whether I gave that impression that I was miserable, but it kind of threw me back a bit. I was like, you are kidding me? Is my persona coming across that bad because I am miserable because I am in pain? So I went ahead and gave it a try. ... It is better if I can try and find an alternative method to control my pain than to keep taking these meds, because they are not very good for my liver. (PT11)

At times, this theme was connected to patients' openness to trying anything to avoid medication/ surgery and/or because neither had been successful.

\section{Openness to Anything}

Many of these patients were willing to try anything because 1) they dealt with chronic ailments for years with diminished wellbeing, and/or 2) they tried numerous treatments/physicians and still suffered. Interestingly, many feared needles and lifestyle constraints but managed to obtain acupuncture.

For instance, the following patient coped with rheumatoid arthritis for more than a decade, trying numerous physicians/treatments all unsuccessfully. She also lost her spouse during this timeframe, which she felt exacerbated the disease. Acupuncture improved her wellbeing:

For me, the state of mind I was in, having unbelievable pain, I was like I will try anything. . . . I cannot handle needles. . . . I hate needles. . . . I knew coming in here I am going to get poked. But my state of mind was, I am desperate. I will try anything. If it will work and help me to get rid of this pain or live a life more pain-free, I will go for it. ... When I was doing the acupuncture and I was feeling better it also affects me, which probably sounds crazy, but to where I concentrate more on taking care of myself because I am feeling better, and by feeling better I want to keep feeling better. So I want to eat foods that are good for me. ... Whether it is the actual acupuncture or just my mind. I do not care which 
one it is. If it makes me be able to enjoy life, I will go for it. (PT1)

\section{Discussion}

The factors and barriers reported by physicians/ patients help illustrate conditions needed to enhance physicians' ability to provide acupuncture and patients' willingness to engage and sustain it. Although there was some overlap in their experiences, different factors were reported more frequently by each group, revealing unique needs that need to be addressed to enhance the potential success of medical acupuncture in a family medicine setting. At the same time, participants' experiences help to illustrate strategic approaches to managing these barriers-strategies that can be used by other individuals/institutions to enhance care delivery and patient engagement. Our findings demonstrate common issues that are likely to occur when other clinics/settings try to implement acupuncture and train current staff, as well as present issues that may arise when physicians move to a new setting/institution.

Patients here described debilitating chronic pain and desperation for a solution as motivation for seeking acupuncture. This echoes findings of Kligler, et $\mathrm{al}^{17}$ that identified patient feelings of desperation as a factor in choosing acupuncture. In our study, this motivation translated to proactive behaviors that can overcome barriers to treatment. Although this may be limited to this sample, it is notable that even highly engaged patients encounter system roadblocks that do not necessarily have to be there.

Patient-reported barriers of limited appointment access are in contrast with those found by Tippens and colleagues ${ }^{20}$. In their community clinic model, patients cited the convenience and ease of scheduling acupuncture treatment as facilitating factors. Those patients felt empowered by the ability to exercise control over when and how they were treated. If family medicine is to integrate acupuncture into the clinical schedule, clinics will need to adapt physician schedules and templates to accommodate for the frequent, patterned nature of acupuncture. One potential method could be openaccess scheduling for acupuncture patients. ${ }^{18}$

Our study extended previously explored patient experiences of acupuncture treatment to include physician perceptions of barriers and facilitators. From that perspective, it is notable that it is phy- sicians who describe patient aversion to needles as a barrier to treatment. Previous studies have demonstrated that patient perception of needles influences their willingness to try acupuncture ${ }^{19}$; however, our results show that physicians actually communicate with patients differently once they know that a patient has an aversion to needles. More research is needed to understand how physicians describe the procedure to patients and if that description influences patient acceptance of the modality.

Physicians also reported systematic factors: limits of equipment, space, personnel, and time. Physicians repeated patient frustrations with appointment access when they discussed time challenges. This finding also replicates the findings of Yeh et $\mathrm{al}^{13,14}$ that time is a barrier to integrating acupuncture into practice. Here, physicians add the perception of limits of patient time as a barrier to regular acupuncture treatment. Beyond time, physicians report the logistic requirements for providing any type of patient care. Adding acupuncture to a family medicine clinic requires more equipment, more space, and more personnel. From this perspective, integrating acupuncture into an existing family medicine clinic will require either the withdrawal of other services or growth in the clinic itself.

Patients in this study represent those who engaged in acupuncture, with both positive results and no perceived result. Future research should include patients who chose not to try acupuncture. This sampling approach could reveal additional or different factors/barriers to trying acupuncture. Three additional systematic features distinguish our sample. First, many physicians were newly trained in medical acupuncture. Results may reflect the novelty of a new skill and its uptake challenges. Second, this medical center exhibits a high saturation of family physicians who are trained in medical acupuncture (17 of 48). Our results demonstrate that even with more than a third of physicians trained, both physicians and patients perceive barriers to practice. Physicians practicing medical acupuncture in less saturated setting may identify more or different barriers than we describe here. Lastly, this clinic is located within a federally funded health system, which provides acupuncture to primary care patients at no additional cost. This system feature could explain why cost of care did not emerge as barrier as it has in previous studies..$^{17,20,21}$ 
The authors acknowledge Daniel Schaeffer and Heather Rider for their support in recruitment and scheduling. Research support was also provided by the Military Primary Care Research Network.

To see this article online, please go to: http://jabfm.org/content/ 31/2/236.full.

\section{References}

1. Niemtzow RC, Burns SM, Cooper J, Libretto S, Walter JA, Baxter J. Acupuncture clinical pain trial in a military medical center: Outcomes. Med Acupunct 2008;20:255-61.

2. McKee MD, Kligler B, Fletcher J, et al. Outcomes of acupuncture for chronic pain in urban primary care. J Am Board Fam Med 2013;26:692-700.

3. Wang SM, Dezinno P, Lin EC, et al. Auricular acupuncture as a treatment for pregnant women who have low back and posterior pelvic pain: A pilot study. Am J Obstet Gynecol 2009;201:e271-279.

4. Coeytaux RR, Befus D. Role of acupuncture in the treatment or prevention of migraine, tension-type headache, or chronic headache disorders. Headache 2016;56:1238-40.

5. Zhang Y, Lin L, Li H, Hu Y, Tian L. Effects of acupuncture on cancer-related fatigue: a meta-analysis. Support Care Cancer 2018;26:415-425.

6. Gould A, MacPherson H. Patient perspectives on outcomes after treatment with acupuncture. J Altern Complement Med 2001;7:261-8.

7. Helms JM. An overview of medical acupuncture. In: Jonas WB, Levin JS, eds. Essentials of complementary and alternative medicine. Baltimore, MD: Williams \& Wilkins; 1999.

8. Sherman KJ, Cherkin DC, Eisenberg DM, Erro J, Hrbek A, Deyo RA. The practice of acupuncture: Who are the providers and what do they do? Ann Fam Med 2005;3:151-8.

9. Crumley ET. Models for integrating medical acupuncture into practice: An exploratory qualitative study of physicians' experiences. Acupunct Med 2016;34:280-9.

10. Lu DP, Lu GP. An historical review and perspective on the impact of acupuncture on U.S. medicine and society. Med Acupunct 2013;25:311-6.
11. Robinson TW. Western acupuncture in a NHS general practice: Anonymized 3-year patient feedback survey. J Altern Complement Med 2012;18: 555-60.

12. Coeytaux RR, Park JJ. Acupuncture research in the era of comparative effectiveness research. Ann Intern Med 2013;158:287-8.

13. Yeh GY, Ryan MA, Phillips RS, Audette JF. Doctor training and practice of acupuncture: Results of a survey. J Eval Clin Pract 2008;14:439-45.

14. Yeh GY, Phillips RS, Davis RB, Eisenberg DM, Cherkin DC. Visit time as a framework for reimbursement: Time spent with chiropractors and acupuncturists. Altern Ther Health Med 2003;9: 88-94.

15. Glaser BG, Strauss AL. The discovery of grounded research: Strategies for qualitative research. New York, NY: Aldine De Gruyter; 1967.

16. Strauss A, Corbin J. Basics of qualitative research: Techniques and procedures for developing grounded theory. Thousand Oaks, CA: Sage; 1998.

17. Kligler B, Buonora M, Gabison J, Jacobs E, Karasz A, McKee MD. "I Felt Like It Was God's Hands Putting the Needles In": A Qualitative Analysis of the Experience of Acupuncture for Chronic Pain in a Low-Income, Ethnically Diverse, and Medically Underserved Patient Population. J Altern Complement Med 2015;21:713-9.

18. Cameron S, Sadler L, Lawson B. Adoption of openaccess scheduling in an academic family practice. Can Fam Physician 2010;56:906-11.

19. Oh B, Eade T, Kneebone A, et al. Factors affecting whether or not cancer patients consider using acupuncture. Acupunct Med 2017;35:107-113.

20. Tippens KM, Chao MT, Connelly E, Locke A. Patient perspectives on care received at community acupuncture clinics: A qualitative thematic analysis. BMC Complement Altern Med 2013;13:293-293.

21. Hopton A, Thomas K, MacPherson H. The acceptability of acupuncture for low back pain: A qualitative study of patient's experiences nested within a randomised controlled trial. PloS One 2013;8(2): e56806. 